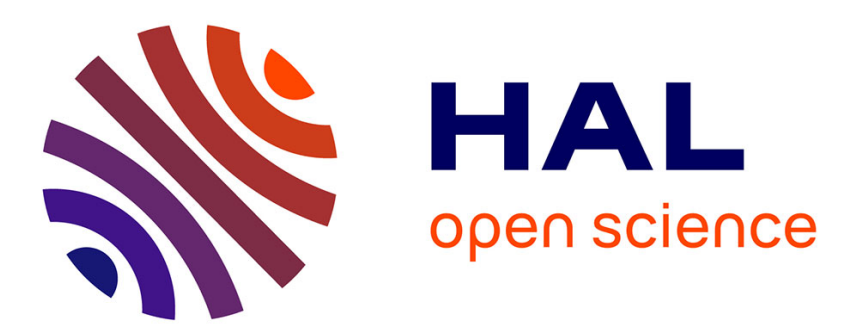

\title{
Les enjeux organisationnels de la qualité : une mise en perspective
}

Franck Cochoy, Gilbert de Terssac

\section{To cite this version:}

Franck Cochoy, Gilbert de Terssac. Les enjeux organisationnels de la qualité : une mise en perspective.

Sciences de la Société, 1999, 46, pp.3-18. hal-00188637

\section{HAL Id: hal-00188637 \\ https://hal.science/hal-00188637}

Submitted on 19 Nov 2007

HAL is a multi-disciplinary open access archive for the deposit and dissemination of scientific research documents, whether they are published or not. The documents may come from teaching and research institutions in France or abroad, or from public or private research centers.
L'archive ouverte pluridisciplinaire HAL, est destinée au dépôt et à la diffusion de documents scientifiques de niveau recherche, publiés ou non, émanant des établissements d'enseignement et de recherche français ou étrangers, des laboratoires publics ou privés. 
Centre d'Étude et de Recherche Technique, Organisation, Pouvoir

Les enjeux organisationnels de la qualité : une mise en perspective

Franck Cochoy et Gilbert de Terssac

Université Toulouse II/CERTOP, UMR CNRS 5044

Maison de la Recherche, 5, allées Antonio Machado, 31058 Toulouse CEDEX, FRANCE

E-mail : cochoy@univ-tlse2.fr ; detersac@univ-tlse2.fr 

En quelques années, les politiques de la qualité ont non seulement été mises en œuvre par un nombre croissant d'entreprises, mais cette mise en œuvre, ses aspects et ses enjeux ont suscité un intérêt grandissant de la part des chercheurs en sciences sociales. De nombreux ouvrages (Gomez 1994 ; Mispelblom 1995, Neuville 1997...), plusieurs numéros spéciaux (Revue Française de Gestion 1995 ; Revue d'Économie Industrielle 1996) et une kyrielle d'articles lui ont été consacrés. La mode (gestionnaire) serait devenue une mode (académique), au point qu'on pourrait se demander si le thème mérite encore examen — si la publication d'un énième numéro spécial sur la question est bien justifiée. La présente parution de Sciences de la Société entend apporter une réponse positive à cette interrogation. La prolifération conjointe des démarches qualité et des études qui lui sont consacrées nous offre l'occasion de réfléchir sur les raisons de cette prolifération même, et donc sur les motifs qui en justifient la poursuite. Pour comprendre la qualité, et les enjeux organisationnels et disciplinaires qui lui sont attachés, nous pouvons désormais prendre un peu de recul, un peu de distance.

Un peu de distance: si l'on regarde la qualité de loin, du point de vue de l'économie d'ensemble, il apparaît de plus en plus que l'intérêt qu'on lui porte accompagne la découverte progressive, par les acteurs économiques, de la face «qualitative» de l'économie, et de son caractère indissociable du versant quantitatif traditionnel: nous le savons désormais, la qualification des produits est le préalable de l'échange économique marchand (Salais 1991), aucun «calcul» économique n'est possible sans une définition stable de ce que l'on comptabilise (Desrosières 1993), de sorte que la qualité apparaît comme l'un des dispositifs institutionnels indispensables à l' « équipement» de la rationalité marchande, et contribue, comme tous les dispositifs de ce genre, à la performation de l'économie comme discipline et comme domaine d'activité (Callon 1998).

Un peu de recul : si l'on regarde la qualité d'aujourd'hui sans oublier celle d'hier, on s'aperçoit qu'elle croise non seulement la tradition du contrôle statistique de la conformité des produits héritée de Shewart (Bayart 1998), et son inscription ultérieure dans une logique de «qualité totale» applicable à l'ensemble des membres de l'organisation (Gogue 1990 ; Dragomir \& Halais 1995), mais aussi le mouvement historique de la normalisation industrielle qui, après avoir concerné l'harmonisation des dispositifs techniques (équipements ferroviaires, matériels électriques, filetages...) s'étend désormais de plus en plus au domaine social, avec la définition de standards en matière d'hygiène, de sécurité, ou de services. Or 
depuis que les démarches qualité ont été reprises et adaptées dans des collections de normes nationales et internationales — dont la série ISO 9000 serait le fleuron (Tamm Hallström 1996) —, la qualité bénéficie de la dynamique propre des normes, c'est-à-dire des externalités de réseau et des effets de «path dependency» ${ }^{1}$. Cette double origine de la qualité a deux conséquences. La première inscrit l'histoire de la qualité dans un mouvement de progressive remontée de la rationalité technique vers le social, au terme duquel l'effort de rationalisation, d'abord appliqué aux produits obtenus en aval, gagne peu à peu les organisations et les hommes qui interviennent en amont. La seconde confère à la qualité le statut d'un dispositif de plus en plus répandu et irréversible ${ }^{2}$. Ces deux conséquences, prises ensemble, nous donnent la mesure du phénomène en cause : ce qui n'était peut-être, au départ, qu' «une mode » de gestion devient, de plus en plus, « un mode» de «gouvernement de l'entreprise » (Gomez 1996), un dispositif organisationnel dont l'extension, la force et la permanence justifient qu'on y apporte une attention renouvelée, approfondie et continue. Précisément : les articles du présent numéro permettent de faire un tour d'horizon des rapports actuels entre «qualité » et «organisation». Ils nous montrent que si l'attention des chercheurs, d'abord focalisée sur le rôle de la qualité dans les relations partenariales, s'oriente de plus en plus sur les effets organisationnels de la qualité, la mise à jour de ces effets a tôt fait de nous ramener vers l'extérieur, et notamment vers la place du «client»dans le «pilotage qualité » des organisations.

\section{Les effets organisationnels de la qualité}

La mise en œuvre des démarches qualité vise d'abord à donner un signal sur le marché : toute politique de la qualité cherche à garantir au client qu'un produit ou qu'un service est conçu et fabriqué selon un mode d'organisation stabilisé : le client est rassuré parce que ce

1. Rappelons que les externalités de réseau désignent le surplus d'utilité que certains biens reçoivent de leur utilisation par un grand nombre d'agents ( $c f$. par exemple, un logiciel de traitement de texte, dont l'intérêt repose au moins pour partie sur la possibilité d'échanger des documents avec autrui); la «path dependency désigne les conséquences temporelles des externalités de réseau: quand les choix antérieurs accroissent l'utilité d'un bien particulier, les choix présents de ce bien deviennent de plus en plus « dépendants du chemin suivi », d'où de possibles effets de blocage (« lock-in ») et d'irréversibilité (Foray 1990).

2. L'irréversibilité des normes de qualité est bien sûre tempérée par leur propre évolution et/ou l'apparition d'alternatives innovantes. Aujourd'hui, les normes ISO 9000 sont confrontées à un nombre de critiques croissant (paperasserie, retombée d'enthousiasme, gestion formelle du dispositif déconnectée de son application concrète [Bonnet 1996]) mais la logique normative se diffracte (une révision est prévue à l'horizon 2000) et se multiplie, notamment avec les normes de management de l'environnement ISO 14 000, un terrain trop récent pour être couvert ici, mais dont la thèse de Thomas Reverdy (1998) montre tout l'intérêt, notamment pour une étude comparative des différents systèmes de management de la qualité. 
qu'il achète est conforme au produit qu'il attend et il à un mode d'organisation censé assurer la qualité du produit. Cette mise sous contrôle de la variabilité locale et sectorielle du process est supposée gommer les effets de différenciation provoqués par des arrangements locaux et des solutions singulières. Mais cette fonction externe de toute politique qualité ne peut se manifester que si un véritable «travail d'organisation» a été réalisé dans la firme : on découvre ici la fonction interne de la qualité, dont la valeur essentielle est organisationnelle. En quoi consiste ce travail d'organisation? Quels en sont les modalités et les enjeux ? Qui en sont les acteurs? Plusieurs communications présentées ici donnent de précieuses indications sur les pratiques touchant la mise en œuvre des démarches qualité ; elles ont en commun de mettre l'accent sur la fonction interne de la qualité et sur sa valeur organisationnelle ${ }^{3}$.

\section{A. La qualité au cour de l'action organisationnelle}

Dire que la qualité est au cœur d'un mouvement organisationnel ne vise pas à limiter son importance, ni à réduire ses effets ; il s'agit plutôt de remettre la qualité à sa place, ou plus exactement de la situer au cœur de l'action organisationnelle dont elle fait partie et dont elle porte la trace. Cette perspective est ancrée dans le champ de la pensée organisationnelle, qui nous donne les instruments conceptuels pour analyser les pratiques d'organisation indissociables de toute démarche qualité. Nous utiliserons les travaux de Simon (notamment son ouvrage Administration et processus de décision [Simon 1983]) pour comprendre la nature de ces pratiques. Organiser c'est «faire faire des choses», étant entendu que les principes et les méthodes qui gouvernent l'action font l'objet de «choix préliminaires », c'està-dire d'une décision préalable. Pour Simon, la construction d'une organisation n'est rien d'autre que « mettre en place un personnel d'exécution et lui superposer une équipe de cadres capables de l' «influencer», afin qu'il agisse de façon efficace et coordonnée » (ibid., p. 4). Notons avec lui que «l'organisation enlève donc à l'individu une partie de son autonomie de décision et lui substitue un processus de décision au niveau de l'organisation. Ordinairement, les décisions que prend l'organisation pour l'individu 1) précisent sa fonction, c'est-à-dire sa compétence générale et la nature de ses devoirs ;2) confèrent l'autorité, c'est-à-dire désignent qui dans l'organisation est investi du pouvoir de prendre de nouvelles décisions pour

3. Bien que les auteurs ne peuvent être tenus pour responsables des interprétations que nous proposons, l'essentiel de l'argumentation avancée leur doit beaucoup. C'est pourquoi lorsque nous l'estimerons nécessaire, nous leur emprunterons quelques propos, tout en gardant notre liberté pour en tirer les enseignements. 
l'individu ; 3) fixent d'autres limites à sa liberté de choix de façon à « coordonner les activités des divers collaborateurs de l'organisation» (p 10). En quoi la qualité est-elle liée à l'action organisationnelle ? Tout porte à croire qu'avec les démarches qualité, les choses changent : elles se présentent d'emblée comme une remise en cause de l'organisation ou tout au moins d'une certaine manière d'organiser. Pourtant, nous voudrions montrer qu'avec les démarches qualité on ne peut pas se défaire de l'action organisationnelle : la qualité apparaît même aujourd'hui comme l'un de ses maillons décisifs, pour trois raisons.

Premièrement, la qualité est un processus de «cadrage » des actions courantes de tous les acteurs impliqués dans la production d'un bien ou d'un service. Les démarche qualité visent à expliciter tout le processus de production, de la conception à la fabrication, en définissant la cible à atteindre et les moyens qui permettent d'y parvenir. Elles participent à la définition des cadres de l'action, de telle sorte que l'action de chacun se situe dans ce cadre explicite : elles se traduisent par des règles d'action visant à confiner les individus et elles s'apparentent en cela à un agir organisationnel. En effet, toute organisation définit ses finalités, ses objectifs ou le résultat désiré, qu'il s'agisse de la continuité de service, du maintien de l'ordre public, de la production de biens. De tels objectifs généraux sont ensuite décomposés et répartis entre des individus et des territoires. Pour réaliser ces objectifs, des moyens sont mis en place pour faire en sorte que les individus se comportent dans le sens attendu par l'organisation. Les moyens d'influence sont conçus pour orienter le comportement des individus et faire en sorte que le subordonné décide dans le sens attendu: d'où la formulation de règles visant à structurer les espaces d'action des individus, règles qui définissent la contribution et la rétribution de chacun, sa place et sa fonction, les méthodes et les procédures qu'il devra mettre en œuvre, mais aussi les modalités de communication et de coordination des individus entre eux. Il est donc clair que le fait d'organiser signifie orienter, influence, «formater », contrôler (au sens anglais) le comportement des individus.

L'action organisationnelle est entendue comme une chaîne de décisions visant à orienter le comportement des multiples acteurs qui interviennent dans le processus ; cette perspective fait de la qualité un maillon essentiel pour étendre l'action organisationnelle à tous les acteurs qui contribuent à la production d'un bien ou d'un service et à tous les stades de cette production. La logique du «confinement» ne se réduit pas aux opérateurs qui se trouvent au bout de la chaîne des accomplissements: elle touche toute la ligne hiérarchique. Un directeur d'établissement ne cherche pas simplement à orienter le comportement de ses subordonnés, 
mais il est lui-même contraint par les décisions prises en amont: celle de la direction du groupe ou de l'administration dont il relève. Un agent intervenant dans la conception du produit ou du procédé et des méthodes de fabrication ne fait pas que définir l'action des autres : il est lui-même contraint par les décisions prises en amont et doit rendre compte de ce qu'il a fait. De plus la «logique de confinement» n'est jamais totale : les individus qui sont « en bout de chaîne » conservent toujours une part de décision et d'initiative, non seulement parce qu'ils peuvent à tout moment menacer de se retirer du processus coopératif (par exemple « désobéir »), mais aussi parce qu'ils ne sont pas réductibles à de simples exécutants. On le voit, les démarches qualité vont prêter main forte au processus de rationalisation des activités productives en étendant l'action d'organiser à tous les acteurs et à tous les stades de l'élaboration et de la réalisation d'un service ou d'un bien.

Deuxièmement, la qualité est un processus de réduction entre «ce qui se fait » et ce qui «doit » se faire. Il n'est pas une recherche dans le domaine des sciences sociales portant sur l'analyse des systèmes de travail qui n'aboutisse au constat d'un écart entre d'un côté les pratiques professionnelles et de l'autre les schémas d'organisation préconisés. Ce constat n'a bien sûr rien de nouveau, puisque à la fin du $19^{\text {ème }}$ siècle les premiers travaux menés dans les entreprises ou les administrations faisaient état de tels décalages. Quel que soit l'univers professionnel, les recherches montrent un écart entre les schémas d'organisation issus des décisions prises en amont et les pratiques réelles. Les règles de sécurité ne sont pas toujours respectées, les délais sont parfois remis en cause, les budgets sont dépassés, les règlements ne sont pas mis en œuvre à la lettre, les directives venant «d'en haut» sont interprétées, les procédures sont adaptées, les instructions sont infléchies, les critères de qualité sont discutés, reformulés, voire redéfinis. L'univers des organisations est peuplé de normes et de règles qui spécifient les buts visés, les contraintes et les modes de légitimation de l'autorité ; celles-ci sont conçues pour orienter, voire pour homogénéiser les pratiques des personnes membres de l'organisation. Néanmoins la réalité du fonctionnement des organisations n'est pas réductible à l'application stricte des instructions, ni même à la bonne utilisation des procédures, comme s'il n'existait qu'une seule rationalité : toute application requiert toujours une interprétation et une adaptation élaborées et mises en œuvre par un acteur collectif qui développe des arrangements et des compromis. Dans cette perspective, on peut dire que les solutions retenues et appliquées par les intéressés dans un contexte organisé ne constituent ni le reflet, ni le décalque des règles formelles: leurs pratiques témoignent d'une part de leurs compétences, de leurs initiatives, de leurs stratégie et d'autre part des limitations du contexte 
organisé, lequel repose non seulement sur une vision incomplète du contexte réel mais aussi sur l'existence d'une concurrence entre les objectifs de l'organisation et ceux que poursuivent ses membres.

En quoi les démarches qualité interviennent-elles pour modifier cet état des lieux ? Leur intervention vise à réduire l'écart entre les pratiques ordinaires et les règles préalables qui visent à les orienter. Certes, il ne s 'agit plus d'éditer des règles «d'en haut», mais de les formuler «par le bas », à partir de la contribution de ceux qui réalisent le travail et qui doivent désormais produire ces règles à l'issue d'une activité d'écriture et de mise en mot de leurs pratiques. Mais cela ne change rien, puisqu'il s'agit quand même d'écrire ce que l'on doit faire, même si ce que l'on doit faire est dérivé de ce que l'on fait effectivement : les auteurs de la construction du cadre changent, mais le cadre des actions n'en a pas moins de force coercitive ; peut-être même cette force coercitive est-elle renforcée du fait de sa plus grande légitimité.

Troisièmement la qualité est un processus de mise en cohérence des actions contrôlé d'en haut, qui pourrait bien marquer un retour de la régulation centralisée et hétéronome. La qualité apparaît comme un maillon essentiel du travail d'organisation dans la mesure où il s'agit d'un processus de cadrage qui vise à conformer les individus, à mettre en cohérence leurs actions et à contrôler le tout d'en haut. La performance d'une entreprise ou d'une administration est liée à la qualité de son organisation: celle-ci dépend certes des actions terminales, courantes et dites « d'exécution » qui permettent (ou non) d'atteindre les objectifs. Mais la performance dépend aussi des actions qui précèdent l'exécution, c'est-à-dire des décisions prises par l'ensemble des acteurs de la ligne hiérarchique et que nous appellerons « actions structurantes ». Les démarches qualité vont permettre, non pas d'opposer les deux formes d'action (structurantes et courantes), mais de les penser ensemble et plus exactement de les mettre en cohérence. Ce qui est au cœur de cette mise en cohérence, ce n'est pas simplement la production de règles plus pertinentes ou plus légitimes, mais bien la régulation entre ces deux ordres. Il nous semble donc opportun de situer les démarches qualité du point de vue de la régulation du processus de production des règles : certes, la production de règles « par le bas » est bien une occasion pour les intéressés de produire des règles autonomes, mais cette production reste contrôlée d'en haut, puisque la régulation du processus de production de règles est extérieure aux intéressés, elle est hétéronome. Il serait donc préférable de parler ici d'espace de discrétion plutôt que d'autonomie, afin de désigner «l'espace d'action où 
l'acteur peut choisir entre des alternatives, mais dans un milieu de dépendance » (Maggi 1993).

\section{B. Les ambiguïtés organisationnelles de la qualité}

On le devine, la qualité est un objet complexe, qui « retravaille » l'organisation comme la compréhension que nous pouvons en avoir : en témoignent la grande variété des comptesrendus et des points de vue rassemblés dans le présent numéros... variété qui nous semble permettre la mise à jour d'(au moins) trois ambiguïtés organisationnelles de la qualité.

La première de ces ambiguïtés intervient dès la mise en œuvre des démarches qualité, sous la forme d'un quiproquo fondateur : d'un côté, l'encadrement adopte la démarche qualité parce qu'il pense qu'un tel dispositif va lui permettre de mieux connaître sa propre organisation et donc de mieux cadrer, contrôler, superviser, stabiliser, enchaîner les gens à leur poste et à leur parole; d'un autre côté la base joue le jeu, parce qu'elle pense que sa participation à l'écriture des procédures va lui donner les moyens de préserver et de légitimer ses acquis, de voir ses compétences reconnues, d'obtenir des garde-fous écrits, etc. Cette première ambiguïté se retrouve dans l'écriture des procédures, qui vise certes la constitution d'un «référentiel commun », le rassemblement d'un savoir organisationnel accessible à tous et partagé par tous, mais qui, pour ce faire, consiste aussi à «séparer» les savoirs des personnes, à dissocier les compétences des individus, au risque de fragiliser leur identité et leur position professionnelle (Dubois \& al.). L'ambiguïté fondamentale des démarches qualité se retrouve enfin dans l'usage des procédures qui, une fois écrites, peuvent ou bien servir de guides et de supports pour l'action - $c f$. la contribution subtile de Sophie Dubuisson, qui montre combien «la délégation aux dispositifs d'une partie de l'action et des responsabilités ouvre aux acteurs de nouveaux espaces d'autonomie et d'action » — ou bien faire office d'instrument de contrôle, non seulement parce que la qualité enchaîne les individus à leurs propres prescriptions, sous la forme de l'autocontrôle (Rot), mais aussi parce que l'évaluation des actions, en dernier ressort, reste le privilège de la seule hiérarchie (Doumenc). D'un côté les démarches qualité permettent à chacun de «dominer la situation » en définissant la place de chacun et en rendant visible l'ensemble du processus ; d'un autre côté, la domination s'entend comme la possibilité, pour la hiérarchie, de contrôler les pratiques des individus via la recherche d'une certaine homogénéisation des pratiques dont la plupart des contributions se font ici l'écho. 
Ces ambiguïtés sont pour une part redevables aux démarcations très particulières que la qualité introduit dans le processus de production des règles : si, dans l'organisation classique, la décision des uns structure l'action des autres, dans l' «organisation qualité », les prescripteurs et les destinataires peuvent être en partie les mêmes et se situer à un moment donné du côté de la décision et à un autre du côté de l'exécution: c'est le cas lorsque l'opérateur devient l'écrivant des procédures qu'il devra appliquer ensuite ${ }^{4}$. Certes, le rapport de subordination qui relie les individus est changé, mais pour un temps seulement ; en effet, il y a bien une procédure de décision qui vise à ne pas décider dans le dos ou à la place des intéressés. Cette dimension politique s'ajoute à la dimension instrumentale : la procédure de décision est avant tout une décision de procédure qui conduit à penser que la définition de la règle n'est plus le monopole de l'encadrement, puisque l'encadré devient provisoirement l'encadrant.

Les démarches qualité visent à créer un référentiel collectif, c'est-à-dire un ensemble de valeurs et d'orientations partagées et un ensemble de normes d'action, afin de remettre de la continuité cognitive et du lien social là où la division du travail avait introduit discontinuités et ruptures. Incontestablement, un climat de normativité distribuée s'instaure, dans lequel la baisse du contrôle (ou sa délocalisation vers le bas) apparaît comme une source de contrôle, au sens où l'encadrement est en quelque sorte contraint de partager sa capacité à produire des règles et des normes avec les destinataires de ces normes. Il s'agit en quelque sorte de remettre dans la boucle organisationnelle ceux qui en avaient été écartés pour procéder à l'explicitation de ce qui était opaque et échappait au contrôle externe ; en même temps, il s'agit aussi de donner la main «en bas» pour faire remonter cette compétence qui accompagne les accomplissements, en explicitant des manières de faire et en les exprimant selon une manière de voir, une codification unique.

Dès lors que les règles d'action sont co-produites, leur dimension prescriptive ne devientelle pas plus impérative ? Les encadrés voient leur autonomie reconnue : ils sont reconnus dans leur capacité de produire leurs propres règles d'action. Ils sont donc en situation d'auto-nomie, c'est-à-dire de production de leurs propres normes d'action. Le paradoxe est que cette auto-nomie est octroyée pour se contraindre, c'est-à-dire pour produire des règles de

4. D'une certaine manière, l'originalité des démarches qualité consiste à introduire une «prescription en kit», puisque l'on demande aux gens d'écrire et de coordonner leurs propres règles d'après le «plan de montage » que représente pour eux le référentiel. 
contrôle. Les destinataires devraient avoir des comportements de loyauté à l'égard de l'organisation d'autant plus forts qu'ils ont participé à la production des règles : la confiance devrait s'imposer dans les dispositifs ; les individus devraient se soumettre aux prescriptions, les appliquer sans discussion, car s'ils ne le faisaient, l'encadrement pourrait le leur reprocher, en invoquant le fait qu'ils ont donné leur accord pour légitimer et valider les contraintes d'action. Les travaux présentés montrent qu'il n'en est rien et que la confiance dans le processus s'accommode d'une méfiance assez grande envers son résultat. En effet, si les acteurs prisent assez généralement l' «émergence de nouveaux espaces de négociation et d'interaction » (Dubois \& al.) qu'ouvre la mise en place des démarches qualité, ils anticipent assez bien les possibilités de contrôle accru que permet la traçabilité des pratiques (Rot). Il en résulte un usage sélectif du dispositif, avec notamment la préservation d'une certaine « opacité » et la préservation de poches d'autonomie (ibid.).

\section{De l'apprentissage à l'intelligence organisationnels}

En dissociant les savoirs des personnes, en organisant le passage des «petits carnets » individuels au «gros carnet collectif » des procédures normalisées (Campinos \& Marquette); la qualité redistribue non seulement les cartes dans l'entreprise (Mounier), mais elle pose finalement la question de l'usage de ces cartes et celle, encore plus cruciale, du sens du jeu.

L'usage des cartes relève d'une dynamique d'apprentissage, et révèle les ambiguïtés qui lui sont attachées. La consignation et la circulation des savoirs sous forme d'écrits redistribuent la connaissance sur l'entreprise dans l'entreprise, et permettent à chacun non seulement d'apprendre ce qu'il fait en l'exposant, mais surtout de l'apprendre aux autres en l'écrivant. Cette opération d'extraversion des savoirs découvre, dépouille et fragilise certains, en supprimant des zones d'incertitude, en clarifiant la place de chacun, ou en portant la menace d'une possible substitution entre personnels (Cochoy \& al. 1998). Mais elle donne aussi des armes au collectif des salariés, en reconnaissant les savoirs acquis, en capitalisant la somme des compétences sur l'organisation, en permettant une formation plus rapide des plus jeunes (Campinos \& Marquette), en déchargeant les acteurs de la renégociation continue des règles du jeu (Dubuisson).

Le sens du jeu apparaît non seulement avec la mise à jour et la transmission des savoirs professionnels, mais plus généralement encore avec toutes les «remontées d'information» 
consécutives à la mise en œuvre du principe de «traçabilité ». Ce principe, en organisant la consignation systématique des actions et des événements de travail (Campinos \& Marquette) ou en sauvegardant le chemin qui permet de remonter de la consommation à la production (Sans \& Fontguyon) encombre l'organisation de toute une série d'inscriptions, de formulaires, de codes qui posent tôt ou tard un problème crucial, celui du sens des informations, de leur exploitation possible, bref, des possibilités d' «intelligence organisationnelle » qu'offre la mise en correspondance des éléments recueillis (Cochoy \& De Terssac 1998).

Pour distinguer le recueil des traces de leur usage réflexif, et pour mieux souligner les enjeux d'une telle distinction, nous avons proposé ailleurs de dissocier la «traçabilité » — la consignation écrite des actions singulières - de la «mappabilité » (de l'anglais to map, cartographier) - l'intelligence des traces, l'exploitation méthodique et réflexive de ce qui est recueilli (ibid.). Les contributions de Michel Dubois ( $\&$ al.) et de Myriam CampinosDubernet et Christian Marquette semblent confirmer l'intérêt d'une telle distinction, les premiers en soulignant «le problème essentiel» que pose «l'interprétation de la codification fournie» et la possibilité de «reconstruire un système d'information interne global et cohérent» à partir de «connaissances [...] auparavant peu explicites et disséminées dans l'organisation »; les seconds en attirant l'attention sur les enjeux d'un usage ponctuel ou systématique des traces.

Si l'on s'en tient à la pure traçabilité, le dispositif de codage apparaît effectivement comme un dispositif de «surveillance», qui permet l'identification occasionnelle des «causes spéciales » (ou pour le dire plus crûment, des opérateurs «coupables ») (Rot), et qui laisse en revanche dans l'ombre les «causes premières », les défauts d'organisation amont qui sont souvent à l'origine des défauts répétés observables en aval. Mais le rassemblement et le recoupement de l'ensemble des traces peut aussi «susciter un mouvement dynamique permettant non seulement de traiter les réclamations, mais de s'attaquer aussi aux défauts mêmes du système »(Campinos \& Marquette). C'est ici que s'opère le passage de la traçabilité à la mappabilité, de l' «enregistrement des pratiques » à la «cartographie des processus » (Boissières \& de Terssac 1998). Cette dernière intervient ex post, lorsque «sont mis en œuvre des dispositifs organisationnels spécifiques (groupes d'amélioration et de progrès, fiches d'anomalies) »et que s'engage «un travail rétrospectif sur le procédé » (Campinos \& Marquette). Mais - la découverte est plus originale - elle intervient aussi ex ante, dès la mise en œuvre de la démarche qualité, dans la mesure où «la mise en trace 
introduit une nécessaire réflexion préalable sur ce qui doit être "donné à voir" », dans la mesure aussi où « [l'écriture des procédures] sélectionne parmi les savoirs pratiques propres à la fabrication ceux qui semblent les plus efficaces, et conduit donc à préalablement identifier les critères de cette efficacité » (ibid.).

Alors que le traçabilité relève assurément du panoptisme (voir sans être vu), la mappabilité définie comme cadrage préalable et comme usage réflexif des traces offre la possibilité d'une intelligence organisationnelle, dont le sens et la destination restent tout entiers à la discrétion des acteurs. S'il n'est bien sûr pas exclu qu'une telle pratique de synthèse des traces alimente un contrôle accru des salariés, il est tout aussi possible qu'elle débouche sinon sur l'ouverture d'un débat général sur l'organisation, du moins sur un accroissement de la vision que chacun a de l'ensemble - ce qui est tout le contraire du panoptisme. L'inscription des actions entre les commandements des procédures et les retours d'information véhiculés par la traçabilité établit un continuum entre la prescription et l'évaluation ; pour la première fois, les démarches qualité opèrent un bouclage continu entre l'action et la règle (Domenc \& Marquié), mais le surcroît de contrôle s'accompagne aussi de gains cognitifs (Dubuisson) qui ne laissent pas de questionner l'organisation dans son ensemble (Dubois \& al.).

\section{Le sens de la qualité : un changement conservateur}

Finalement, le sens de la qualité repose autant dans sa lettre que dans ses mises en œuvre particulières. Le coup de génie de la qualité consiste à établir un processus de changement éminemment conservateur : la qualité vise paradoxalement à conserver l'organisation pour mieux la changer, à subordonner l'amélioration du process à l'écriture de l'organisation existante. En exigeant que chacun écrive ce qu'il fait et fasse ce qu'il a écrit, en ordonnant aux salariés «faîtes ce que vous faîtes !», la qualité invente une régulation inédite qui consiste à « prescrire l'autonomie », et à transformer cette dernière en « autocontrôle » (Rot 1998). Tous les paradoxes, toutes les ambiguïtés, toutes les potentialités et toutes les difficultés de la qualité reposent finalement sur cette injonction bizarre qui, en apparence, est tout entière empreinte d'une vaine redondance mais qui, en réalité, est porteuse d'implications organisationnelles insoupçonnées. Souvent, la qualité instaure un jeu de dupe, parce que chacun se trompe au départ sur le sens du dispositif. L'encadrement croit qu'il tient là le moyen de mieux contrôler les acteurs en les enchaînant à leurs propres pratiques, mais il oublie de voir que le dispositif de contrôle produit aussi des informations et des compétences 
susceptibles de le contraindre en retour. Lorsqu'il s'en aperçoit et comme il garde encore la maîtrise, il s'arrête bien souvent de «jouer»: il ignore les remontées d'information qui désignent ses propres carences. Les subordonnés quittent aussi la partie, pour des raisons exactement symétriques : ils ne mettent plus à jour les procédures parce qu'ils s'aperçoivent qu'elles ne servent à rien sinon à codifier leurs savoirs, et parce qu'ils voient bien que personne n'utilise les informations qu'ils font remonter (Cochoy \& al. 1998) sinon pour instruire des procès ad hominem (Rot). Il s'ensuit une frustration d'autant plus dommageable qu'elle engage tous les acteurs de l'organisation — des acteurs désormais pleins de savoirs organisationnels partagés, des acteurs à qui «on ne la fait plus », parce qu'ils ont beaucoup appris les uns et des autres. Mais observons combien l'apparition de telles frustrations dérive moins de la qualité elle-même que des ratés de sa mise en œuvre, et de la méconnaissance de ses implications profondes, notamment en termes de « mappabilité » des pratiques.

\section{La qualité « au nom du client », ou comment le marché entre dans l'organisation}

Reste une question, et d'importance. «Au nom de qui » les démarches qualité sont-elles adoptées ? Selon l'identité et le poids du commanditaire, la qualité pourrait bien continuer à s'imposer, indépendamment des vicissitudes organisationnelles de sa mise en pratique. Partout où elle est adoptée, la justification ultime de la qualité est celle du marché, de la concurrence, du «client». Si à l'origine de la qualité organisationnelle on trouve bien la qualité du produit (Sans \& Fontguyon), derrière le produit, on a toujours mis en avant celui qui le consomme ou qui le commande, à savoir le consommateur ou son porte-parole : ce client «donneur d'ordre » qui prescrit autant l'adoption de la démarche qualité que la fabrication proprement dite. Voilà sans doute pourquoi les études de la qualité se sont d'abord intéressées au rôle croissant des référentiels correspondants dans la relation client-fournisseur pour découvrir ensuite leurs implications sociales dans l'organisation, et voilà aussi pourquoi elles reviennent de plus en plus, pour comprendre ce qui se passe en interne, au rôle du client dans l'argumentation et dans les pratiques de la qualité, comme en témoignent les quatre contributions du présent numéro consacrées à ce thème. Les auteurs nous montrent que si la figure du client est la plupart du temps largement rhétorique (Neuville), sa prégnance effective n'est pas à exclure (Mounier) de sorte que la conjugaison de ces deux aspects du client - client virtuel et client réel - mérite sans aucun doute un examen particulier (Louppe, Lecomte et Simbille). 


\section{A. L'autorité plurielle d'un client multiple}

Jean-Philippe Neuville s'interroge sur l'introduction de la figure a priori exogène du « client» comme façon de retravailler les modes de coordination dans l'entreprise. Pour ce faire, il passe en revue les différents types d'intervention du client dans le discours sur la qualité : le client à la place duquel chacun se met, le client-patron de l'entreprise, le clientunité de l'entreprise qui s'adresse au fournisseur-autre-unité de l'entreprise, le client-service de la qualité totale, qui gère l'ensemble du dispositif. L'examen de chacune de ces figures débouche sur la mise à jour d'un paradoxe, d'une impasse et d'une usurpation. Le paradoxe veut que « le client réel, celui qui achète le produit sur le marché, n’est jamais présent dans le site de production». L'impasse est celle de la transposition du modèle marchand dans l'organisation via l'application des relations de type client-fournisseur entre unités d'une même entreprise. Cette transposition est une pure fiction, puisqu'un client n'a de pouvoir que s'il peut choisir entre plusieurs fournisseurs, ce qui n'est pas le cas pour les différentes unités d'une chaîne de montage. L'usurpation est donc celle de la place du client elle-même, qui sert d'abri à l'autorité du chef: derrière chacun des clients se cachent en fait d'autres «clients »... dont on constate que certains ont plus raison que les autres, en proportion de leur place dans la hiérarchie. Identifier la hiérarchisation implicite des clients mis en scène, c'est retrouver l'organisation, la subordination, le contrôle.

Mais une question se pose néanmoins : l'évocation du client consiste-t-elle uniquement à mobiliser un leurre pour masquer « une tradition devenue gênante » - celle de l'autorité ou ne consiste-t-elle pas aussi, pour ceux qui s'en prévalent, à mettre quelqu'un de plus dans leur propre camp ? L'enjeu de fond est bien celui-là : avec le client, l'autorité réelle — en personne - se retrouve diluée dans une figure extérieure, familière, à laquelle chacun s'identifie volontiers parce que nous sommes tous clients, alors qu'inversement nous ne sommes pas tous responsables ou managers. S'il s'agit de se mettre à la place de l'autre, il ne s'agit pas de se mettre à la place de n'importe lequel; avec le client, on n'a pas affaire à une pure machination; derrière les clients figurés se trouvent des clients bien réels. C'est là le virage fondamental : grâce au client, il n'y a plus de fausse conscience, d'illusion, de mensonge, de construction, d'invention, parce que ce qui est construit est aussi saisi, existe bel et bien, sur le marché. Le client permet d'obtenir à la fois la contrainte et la dilution de cette dernière dans une instance extérieure à l'entreprise et souvent insaisissable. 
Plusieurs contributions documentent cette prégnance empirique du client, qui se manifeste soit via les retours d'information qu'assurent les études marketing (Dubuisson), les pratiques de segmentation (Lecomte \& Simbille) et/ou le traitement des réclamations (Campinos \& Marquette) associés à la gestion de la qualité, soit par « le développement des visites clients, la multiplication des audits qualité et plus généralement le développement de la gestion de la qualité » (Mounier). Ces procédures finissent, de proche en proche, par porter les exigences du client et du marché jusqu'au cœur de l'organisation : « "le client au centre” est bien plus qu'une simple figure de rhétorique pur les opérateurs, ces derniers ont de plus en plus conscience que leur entreprise est mortelle, que sa pérennité ne va pas de soi »(ibid.). Le client, avec sa dimension personnelle et charnelle, change la fatalité d'un marché global et abstrait en action possible sur les personnes qui «tiennent » ce marché ; mais ce faisant, une fatalité en remplace une autre : on redevient acteur sur le marché, mais un acteur contraint par les désirs de la clientèle.

En fait, les deux formes du client comme figure de rhétorique ou comme personne empirique sont indissociables, comme le montre Peggy Louppe, qui dresse le constat de « dédoublement du client» en «deux faces »: un «client “formel” » et un «client "concret" ». Si le client formel commande la mise en œuvre des démarches qualité et le respect des procédures qui lui sont associées, le client concret exerce souvent des demandes intempestives, brouillonnes et discrétionnaires. Ce double jeu du client a partie liée à l'une des ambiguïtés fondamentale des dispositifs d'assurance qualité, lesquels proclament en même temps le dogme du «client roi » et celui du «respect des procédures ». Cependant, cette ambiguïté n'est pas forcément négative, dans la mesure où elle sert de base à la recherche d'arrangements négociés entre le client réel et son fournisseur, l'un se prévalant de son « autorité » de « client formel », l'autre lui rappelant les contraintes d'une règle qu'il lui a par ailleurs imposée (Louppe).

\section{B. La place du client}

En fait cette ambiguïté repose tout entière sur la place du client dans la «boucle » de l'assurance qualité : selon que le client est «dehors » ou «dedans », extérieur ou partie prenante dans la démarche qualité, son pouvoir n'est évidemment pas le même discrétionnaire dans un cas — comme nous venons de le voir — beaucoup plus limité dans le second - comme le montre l'application du principe de «coproduction » de la qualité dans le 
secteur des services. Le travail d'Isabelle Lecomte et Jocelyne Simbille fait apparaittre le paradoxe suivant : alors que dans le secteur privé, la qualité est généralement prescrite par un client qui reste prudemment «en dehors » de la «boucle» qu'il impose à tous les autres, la même qualité se trouve, à l'ANPE, imposée au client, à l'usager, qui est placé dans la boucle d'office et un peu malgré lui. L'idée de coproduction consiste à faire de l' «usager » un « partenaire » du service qui lui est rendu, ou plutôt à l'associer à la production d'un service qu'il doit finir par se rendre. L'application de cette logique à l'ANPE se traduit par des résultats pour le moins curieux : «plus le service est coproduit plus le client est satisfait par le processus, indépendamment du résultat». Même si les auteurs ne vont pas jusque-là, leur travail semble montrer que la coproduction consiste à associer le client à l'éventuel échec du service, dont il devient co-responsable, voire à faire passer cet échec pour un succès, en substituant les moyens à la fin, en remplaçant l'ancienne finalité du service — trouver un emploi - par un nouvel objectif purement relationnel - maintenir le contact entre le demandeur d'emploi et l'agence censée lui venir en aide.

Le pouvoir du client donneur d'ordre comme les déboires de l'usager coproducteur de service soulignent combien la performance est importante dans l'appréciation des démarches qualité. Presque toujours, le client apparaît comme l'incarnation «micro » du marché, qui impose ses rythmes, ses incertitudes et ses contraintes au salarié (Mounier) comme à l'organisation (Sans \& Fontguyon). L'exemple de la crise de la vache folle est de ce point de vue particulièrement éclairant. La défection bien réelle des consommateurs sur le marché de la viande bovine a été l'occasion, pour les acteurs de la filière concernée, de mettre le client et la «qualité » au premier rang, et de redéfinir, «au nom du client », l'ensemble des relations partenariales dans le secteur. En se faisant porte-parole des consommateurs, la grande distribution est parvenue à imposer une gestion «à livre ouvert» à l'ensemble de ses partenaires, pour que tout — la qualité et les coûts — soit désormais transparent. Une telle politique se traduit, une fois encore, par des résultats ambigus, puisqu'elle permet à la fois une répartition équitable des chocs exogènes sur la filière de production mais aussi l'enchaînement très étroit des partenaires au distributeur, dont les marges ne sont pas inclues dans la boucle de la transparence (ibid.).

Avec la qualité, les enjeux extérieurs (marchands) sont désormais indissociables des enjeux intérieurs (organisationnels), ne serait-ce qu'au travers des pratiques d'évaluation (Domenc) qui simulent et/ou qui institutionnalisent le regard extérieur de la tierce partie, du client ou du 
marché, et qui relativisent donc sinon le poids, du moins la responsabilité de l'autorité locale, en lui substituant/superposant un nouvel ordre, plus global et plus diffus : l'ordre de la « qualité », du «marché mondial » et/ou de la «clientèle ». Ce qui vaut pour l'organisation dans son ensemble vaut bien sûr pour l'ensemble de ses compartiments. Par exemple, l'introduction de l'approche client inhérente aux démarches qualité change la place habituelle de la maîtrise, comme le montre bien la contribution de Gwenaële Rot : grâce à la qualité, le contremaître peut changer de rôle; il abandonne au dispositif gestionnaire ses fonctions habituelles de « garde chiourme », ce qui lui permet de se rapprocher de la base, d'endosser le rôle beaucoup plus gratifiant et confortable d'intercesseur, de négociateur, de médiateur, de protecteur, de modérateur : la maîtrise se fait complice d'un «management clandestin », en négociant la correction discrétionnaire des excès d'un système devenu aveugle contre une confiance et une coopération accrue des opérateurs. Enfin, ce qui vaut pour les compartiments internes de l'organisation se retrouve aussi au dehors : à l'interface de l'organisation et du marché, les rigueurs du dispositif ouvrent elles aussi un espace de négociation symétrique avec le «client réel », lequel se montre beaucoup plus accommodant sur la qualité lorsqu'on va le voir «en personne » et ferme les yeux sur les non conformités, de sorte que l'invention et le maintien de zones d' «opacité » à la périphérie des démarches qualité se retrouve non seulement en aval, dans les ateliers (Rot), mais aussi en amont, sur le marché (Louppe).

\section{Conclusion}

Finalement, la qualité a au moins le mérite de s'offrir comme une navette commode pour glisser du local au global, de l'organisation au marché, et donc pour comprendre les enjeux de leur articulation. Toutes les contributions réunies ici montrent que lorsqu'un même objet vient traverser toute une série d'organisations, il nous donne les moyens de saisir à la fois comment l'ordre global se diffracte en autant de cas particuliers (Segrestin 1997), et pourquoi les cas particuliers relèvent toujours d'une dynamique plus générale, de sorte qu'avec la qualité, « un cas n'est jamais un cas » (Cochoy \& al. 1998). Le moindre des paradoxes de la qualité n'est-il pas qu'en imposant une logique et des dispositifs communs à un grand nombre de sites (Campinos \& Marquette), il amène les acteurs à raisonner «toutes organisations égales par ailleurs », et donc à retrouver, pour se différencier et sortir vainqueurs de la dure loi du marché, cette dimension humaine qu'ils semblaient abandonner? C'est du moins ce que suggère ce témoignage encourageant recueilli par Peggy Louppe: «du fait que les organisations sont les mêmes, le critère déterminant, ce sont les hommes ». 


\section{Références}

BAYART, D. (1998), « Statistique mathématique et gestion de la qualité, Recherches sur l'histoire de la cognition dans l'organisation industrielle », Cahiers du Centre de Recherche en Gestion, ${ }^{\circ}{ }^{\circ}$ 14, mai.

BoIssieres, I. DE TERsSAC, G. (1998), La robustesse organisationnelle, Rapport d'étude, France Télécom/CERTOP-CNRS, Toulouse.

BONNET, E. (1996), «Les “visions indigènes" de la qualité. À propos de l'appropriation de la démarche qualité dans l'industrie », Revue d'Économie Industrielle, $\mathrm{n}^{\circ}$ 75, 1er trimestre, pp. 77-94.

CAllon, M. (dir.) (1998), The Laws of the Markets, London, Blackwell.

Cochoy, F., Garel, J.-P. \& De Terssac, G. (1998), «Comment l'écrit travaille l'organisation: le cas des normes ISO $9000 »$, Revue française de sociologie, Vol. 39, n 4, pp. 673-699.

Cochoy, F. \& De Terssac, G. (1998), « Traçabilité et mappabilité : deux enjeux qui travaillent l'organisation», 2ème colloque langage et travail, 24-25-26 septembre, Centre de Recherche en Gestion, École Polytechnique, Paris (texte à paraitre in Travail d'organisation, De Terssac, Gilbert (dir.), ouvrage en préparation).

Desrosieres, A. (1993), La politique des grands nombres, Histoire de la raison statistique, Paris, La Découverte.

Dragomir, R. \& Halais, B. (1995), Petite histoire de la qualité, Ministère de l'Industrie, Direction générale des stratégies industrielles, Sous-direction de la Qualité pour l'Industrie et de la Normalisation.

Foray, D. (1990), «Exploitation des externalités de réseau versus évolution des normes: les formes d'organisation face au dilemme de l'efficacité dans le domaine des technologies de réseau », Revue d'Économie Industrielle, $\mathrm{n}^{\circ}$ 51, 1er trimestre, pp. 113-140.

Gogue, J.-M. (1990), Les six samouraïs de la qualité, Les hommes qui ont fondé le management moderne, Paris, Economica.

GoMEZ, P.-Y. (1994), Qualité et théorie des conventions, Paris, Economica.

GOMEZ, P.-Y. (1996), Le gouvernement de l'entreprise. Modèles économiques de l'entreprise et pratiques de gestion, Paris, InterEditions.

MAGGI, B. (1993), « Tradizione e innovazione nello studio interdisciplinare del lavoro », Introduction à l'édition italienne de G. de Terssac, Come cambia il lavoro; efficacia, autonomia, valorizzazione delle competenze. Milano, Etas Libri, pp. 1-28.

MisPelblom, Frederick (1995), Au-delà de la qualité. Démarches qualité, conditions de travail et politiques du bonheur, Paris, Syros.

NeuviLLE, J.-P. (1997), Le modèle japonais à l'épreuve des faits, Paris, Economica.

REVERDY, T. (1998), L'invention du management environnemental, extension de la qualité industrielle et régulation négociée de l'environnement, thèse de doctorat de l'Université Pierre Mendès France, Grenoble.

REVUE D'ÉCONOMIE INDUSTRIELLE (1996), numéro spécial Normalisation et organisation de l'industrie : approches pluridisciplinaires, Ravix, Joël Thomas \& Romani, Paul-Marie (dir.), Revue d'Économie Industrielle, $\mathrm{n}^{\circ} 75,1 \mathrm{er}$ trimestre.

Revue Française de Gestion (1995), dossier Gérer par les normes, Revue Française de Gestion, n 106, novembre-décembre.

Rot, G. (1998), « Autocontrôle, traçabilité, responsabilité », Sociologie du Travail, vol. 40, n 1, pp. 5-20.

SALAIS, R. (1991), « Approches économiques et historiques récentes du marché », Genèses, pp. 162-170.

SEGRESTin, D. (1997), «L'entreprise à l'épreuve des normes de Marché. Les paradoxes des nouveaux standards de gestion dans l'industrie », Revue Française de Sociologie, Vol. 38, pp. 553-585.

Simon, H. A. (1983), Administration et processus de décision, Paris, Economica [1945].

TAMm Hallström, K. (1996), «The production of management standards », Revue d'Économie Industrielle, $\mathrm{n}^{\circ} 75,1 \mathrm{er}$ trimestre, pp. 61-76. 